\title{
Toxicidad hepática causada por medicamentos: revisión estructurada
}

\section{Structured Literature Review of Hepatic Toxicity Caused by Medicines}

Alejandra Cano P., ${ }^{1}$ Laura Cifuentes P., ${ }^{2}$ Pedro Amariles, PhD. ${ }^{3}$

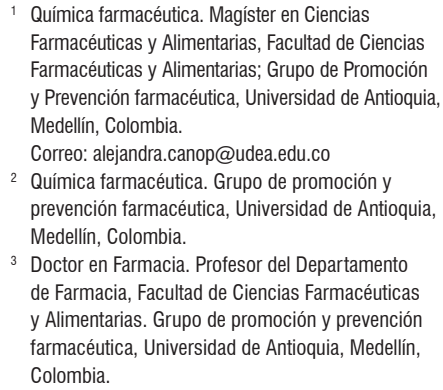

3 Doctor en Farmacia. Profesor del Departamento de Farmacia, Facultad de Ciencias Farmacéuticas y Alimentarias. Grupo de promoción y prevención farmacéutica, Universidad de Antioquia, Medellín, Colombia.

Fecha recibido: $13-10-16$ Fecha aceptado: 06-10-17

\begin{abstract}
Resumen
Objetivos: elaborar un listado actualizado de medicamentos causantes de hepatotoxicidad e identificar, de acuerdo con la evidencia científica, los medicamentos con mayor probabilidad de causar hepatotoxicidad. Método: se realizó una búsqueda en PubMed/Medline utilizando términos Mesh: "liver disease" y "druginduced liver injury". La búsqueda se filtró por: reportes de casos, revisiones, ensayos clínicos, metaanálisis y cartas, hasta diciembre de 2015, en inglés, español y francés. Se incluyeron artículos con evidencia de hepatotoxicidad causada por medicamentos y referencias relevantes; fueron excluidos artículos sin relación con los objetivos de la búsqueda, relacionados con hepatotoxicidad por agentes diferentes, concernientes a otras causas de enfermedad hepática o relacionados con ensayos predictivos o células madre. Algunos aspectos de los medicamentos hepatotóxicos fueron: aparición de hepatotoxicidad, tipo de lesión, mecanismos de hepatotoxicidad, factores de riesgo y manifestaciones clínicas. Para valorar la probabilidad de aparición de hepatotoxicidad y del tipo de lesión se establecieron 3 categorías: definida, probable y posible. Resultados: se identificaron 610 artículos de los cuales se eligieron 402, se excluyeron 208 artículos. Se elaboró un listado con 181 medicamentos y 17 formas farmacéuticas combinadas o regímenes terapéuticos con probabilidad de causar hepatotoxicidad; de estos, 6 medicamentos tuvieron probabilidad definida (metotrexato, minociclina, vancomicina, everolimus, isoniazida y tamoxifeno). Conclusiones: se identificaron más de 180 medicamentos hepatotóxicos, 6 tienen una probabilidad definida, mientras que para la mayoría es posible. La consolidación de la información demostró que diversas categorías de medicamentos tienen mayor probabilidad de ser causantes de hepatotoxicidad.
\end{abstract}

\section{Palabras clave}

Enfermedad hepática, daño hepático inducido por medicamentos.

\section{INTRODUCCIÓN}

La hepatotoxicidad es la lesión causada por la exposición a un medicamento u otros agentes no farmacológicos. La idiosincrasia, edad, género, consumo de alcohol, tabaquismo, uso concomitante de otros fármacos, enfermedad hepática previa o subyacente, factores genéticos y ambientales se consideran factores de riesgo (1-3). Aunque la mayoría de los fármacos lipofílicos podría causar hepatotoxicidad (4), los antibióticos, antiinflamatorios no esteroideos (AINE) y anticonvulsivantes son los grupos farmacológicos con una mayor probabilidad (1, 5-9). Además, entre los medicamentos administrados por vía intravenosa, los antibióticos y antineoplásicos son los grupos más asociados con toxicidad hepática (10). La hepatotoxicidad puede clasificarse en 2 tipos:

1. Reacciones intrínsecas: predecibles, dependientes de la dosis, reproducibles, pero con información limitada de su frecuencia de presentación; y 


\begin{abstract}
Objectives: The aim of this study was to prepare an updated list of drugs that cause hepatotoxicity and identify drugs most likely to cause hepatotoxicity according to scientific evidence. Method: A search of PubMed/ Medline was conducted using the MeSH terms: "Liver disease" and "Drug-induced Liver Injury". The search was filtered by case reports, reviews, clinical trials, metaanalyses and letters until December 2015. The search was limited to articles in English, Spanish and French. Articles with evidence of hepatotoxicity caused by medications and relevant references were included. Articles not related to the objectives of the search were excluded. These include articles related to hepatotoxicity due to other agents, articles about other causes of liver disease and/or articles related to predictive tests or stem cells. Some aspects of hepatotoxic drugs were appearance of hepatotoxicity, type of injury, mechanisms of hepatotoxicity, risk factors and clinical manifestations. Three categories, definite, probable and possible, were established to assess probability of hepatotoxicity and type of lesion. Results: Six hundred ten articles were identified, 402 articles were chosen, and 208 articles were excluded. A list was prepared with 181 drugs and 17 combined pharmaceutical forms or therapeutic regimens likely to cause hepatotoxicity. Of these, methotrexate, minocycline, vancomycin, everolimus, isoniazid, and tamoxifen were categorized as definite probabilities. Conclusions: More than 180 hepatotoxic drugs were identified, six were categorized as definite probabilities, and most were categorized as possibilities. The consolidation of information shows that diverse categories of drugs are likely to cause liver toxicity.
\end{abstract}

\title{
Keywords
}

Liver disease, drug-induced liver damage.

2. Reacciones idiosincrásicas (inmunes o metabólicas): impredecibles, no dosis-dependiente, no reproducibles y afectan una pequeña proporción de pacientes (entre $1 / 1000$ y $1 / 100000$ pacientes expuestos) (11-17).

La hepatotoxicidad intrínseca es menos común, mientras que la idiosincrásica es la más asociada con este evento adverso (12, 18-20). La histología hepática es la herramienta ideal para definir el patrón de toxicidad hepática; sin embargo, en la práctica clínica, la mayoría de las lesiones hepatotóxicas se clasifica de acuerdo con pruebas bioquímicas (21). Según el consenso internacional del Council for Internacional Organizations of Medical Sciences (CIOMS), se considera daño hepático si las enzimas hepáticas se aumentan en más de 2 veces el límite superior normal (LSN); por su parte, el tipo de lesión generada se clasifica en $(12,22,23)$ :

- Hepatocelular: incremento aislado de alanino aminotransferasa $(\mathrm{ALT})>2$ veces el LSN o una relación, entre ALT/ fosfatasa alcalina (FA) $>5$. La ley de Hy considera este tipo de lesión con valores de ALT $>3$ veces el $\operatorname{LSN}(24,25)$.

- Colestásica: incremento aislado de FA >2 veces el LSN o una relación $<2$.

- Mixta: ALT y FA $>2$ veces el LSN y una relación $>2$, pero $<5$.

La hepatotoxicidad se relaciona con disfunción mitocondrial, por inhibición de la respiración celular o alteración en la oxidación $\beta$ de los ácidos grasos $(26,27)$, lo que genera apoptosis, necrosis, autofagia y, con ello, muerte celular $(28,29)$. Las principales manifestaciones clínico-patológicas de la hepatotoxicidad y sus hallazgos histológicos son:

a. Hepatitis aguda (caracterizada por inflamación parenquimal, necrosis y células de Kupffer en los sinusoides);

b. Hepatitis crónica (fibrosis);

c. Hepatitis fulminante (necrosis e inflamación);

d. Hepatitis colestásica (inflamación y daño hepático);

e. Colestasis (tapones biliares en zona 3 );

f. Síndrome de desvanecimiento de conductos biliares (daño en conductos biliares, colestasis e inflamación);

g. Hepatitis granulomatosa (granulomas en tractos portales o parénquima);

h. Esteatosis macrovesicular (gotas de lípidos en el citoplasma del hepatocito);

i. Esteatosis microvesicular (diminutas gotas de lípidos en el citoplasma del hepatocito); y

j. Esteatohepatitis (esteatosis, inflamación lobular, hepatocitos englobados y fibrosis pericelular) $(12,29-31)$.

Estas manifestaciones se acompañan de signos y síntomas inespecíficos como fiebre, fatiga, náuseas, dolor abdominal, ictericia, orina oscura, prurito, ascitis, encefalopatía y aumento de transaminasas $(16,32,33)$.

Aunque unos 1100 fármacos, excluidas sustancias de abuso y productos naturales, se han asociado con hepatotoxicidad (19); la identificación de este evento adverso es un proceso complejo. Por esto, se requiere de una minuciosa indagación, orientada a identificar cualquier sustancia y a descartar otras causas de hepatopatía $(3,8,34)$. 
Además, la biopsia hepática es fundamental en la identificación de la hepatotoxicidad (35). Por su parte, la relación cronológica, entre la exposición al agente sospechoso y la reacción hepatotóxica, es clave. Para establecer la probabilidad de que un fármaco esté asociado con hepatotoxicidad, se han desarrollado escalas clínicas, como la Roussel Uclaf Causalita Assessment Method (CIOMS/RUCAM) y la Clinical Diagnostic Scale o Maria-Victorino Scale (M\&V CDS). Se considera que la escala CIOMS/RUCAM, por su validez de contenido y de criterio, es la más adecuada y genera resultados compatibles con el juicio médico y la opinión de expertos sobre hepatotoxicidad; sin embargo, debido a lo dispendioso de su aplicación, su utilidad en la práctica clínica es limitada (36-38).

El tratamiento de la hepatotoxicidad, debido a la ausencia de una farmacoterapia específica, se fundamenta en suspender el medicamento sospechoso, tratar los síntomas y realizar seguimiento a las pruebas de laboratorio (39). Sin embargo, la utilización de $\mathrm{N}$-acetilcisteína como antídoto para toxicidad por acetaminofén (o en hepatotoxicidad por fenitoína y carbamazepina), o de carnitina para la toxicidad por ácido valproico son algunas excepciones (40).

Un listado actualizado de medicamentos hepatotóxicos y factores asociados podría contribuir a optimizar la identificación y prevención de este evento adverso. Por ello, los objetivos de la revisión fueron: elaborar un listado actualizado de medicamentos asociados con hepatotoxicidad e identificar, de acuerdo con la evidencia científica, los medicamentos con mayor probabilidad de causar hepatotoxicidad. Adicionalmente, sistematizar y precisar información clave como tipo de lesión y probabilidad de ocurrencia, mecanismos fisiopatológicos, manifestaciones clínicopatológicas, variación en las cifras de las enzimas hepáticas, reacción intrínseca o idiosincrásica, factores de riesgo y el desenlace del cuadro.

\section{MÉTODO}

\section{Búsqueda bibliográfica}

Se realizó una búsqueda en PubMed/Medline utilizando los términos Mesh: "liver disease" (drug effects, injuries, patho$\log y$ ) y “drug-induced liver injury”. La búsqueda se filtró por: artículos con palabras clave en el título o resumen, publicados hasta diciembre de 2015 en inglés, español y francés, con acceso al texto completo, que fuesen reportes de casos, revisiones, revisiones sistemáticas, ensayos clínicos, ensayos clínicos controlados, ensayos clínicos aleatorizados, metaanálisis y cartas al editor. Los artículos con evidencia de hepatotoxicidad solo por medicamentos y aquellos considerados relevantes para el tema se incluyeron. Por su parte, se excluyeron los artículos sin relación con los objetivos de la búsqueda, relacionados con hepatotoxicidad por otras sustancias (productos naturales, suplementos dietarios, sustancias de abuso y sustancias industriales), los concernientes a otras causas de enfermedad hepática y los relacionados con ensayos predictivos de hepatotoxicidad o células madre.

\section{ANÁLISIS DE LA INFORMACIÓN}

La elegibilidad de los artículos y la extracción de la información se realizaron por 2 revisores independientes y las discrepancias se resolvieron por un tercer revisor. Se registró, en una base de datos (Excel 2010 para Windows'), el título, autor, año de publicación, tipo de estudio, grupo farmacológico relacionado y cumplimiento de criterios de inclusión para cada una de las referencias encontradas. Además, para los medicamentos hepatotóxicos encontrados se tabuló el grupo farmacológico, código ATC (clasificación Anatómica, Terapéutica, Química, por sus iniciales en inglés), probabilidad de ocurrencia o aparición de hepatotoxicidad, tipo de lesión, probabilidad de aparición del tipo de lesión, mecanismos de hepatotoxicidad, factores de riesgo y manifestaciones clínicas, manejo, desenlace, cifras de enzimas hepáticas y dosis de medicamento. Para los datos numéricos, como los valores de enzimas hepáticas (aspartato aminotransferasa [AST], ALT, FA y bilirrubina total $[\mathrm{BT}]$ ) y los valores de la dosis de medicamento administrado, se calcularon el promedio y la desviación estándar.

\section{VALORACIÓN DE LA APARICIÓN DE HEPATOTOXICIDAD Y TIPO DE LESIÓN}

La valoración de la aparición de hepatotoxicidad y del tipo de lesión se basó en la probabilidad de su ocurrencia (41). Se estableció en 3 categorías, de acuerdo con la evidencia encontrada:

a. Definida: evidencia en metaanálisis, revisiones sistemáticas o ensayos clínicos (aleatorizados o no);

b. Probable: estudios analíticos o descripción en 3 o más reportes de casos clínicos; y

c. Posible: menos de 3 casos reportados o por recomendaciones de grupos de expertos (41).

En el caso de medicamentos que presentaron varias referencias con tipos de estudio diferentes, se utilizaron los de mayor nivel de evidencia.

\section{RESULTADOS}

Se identificaron 610 artículos en la búsqueda, se eligieron 402 que cumplían con los criterios de inclusión, se excluyeron 208 y se incluyeron 46 artículos considerados relevantes para la revisión (Figura 1). Se elaboró un listado con 181 medica- 


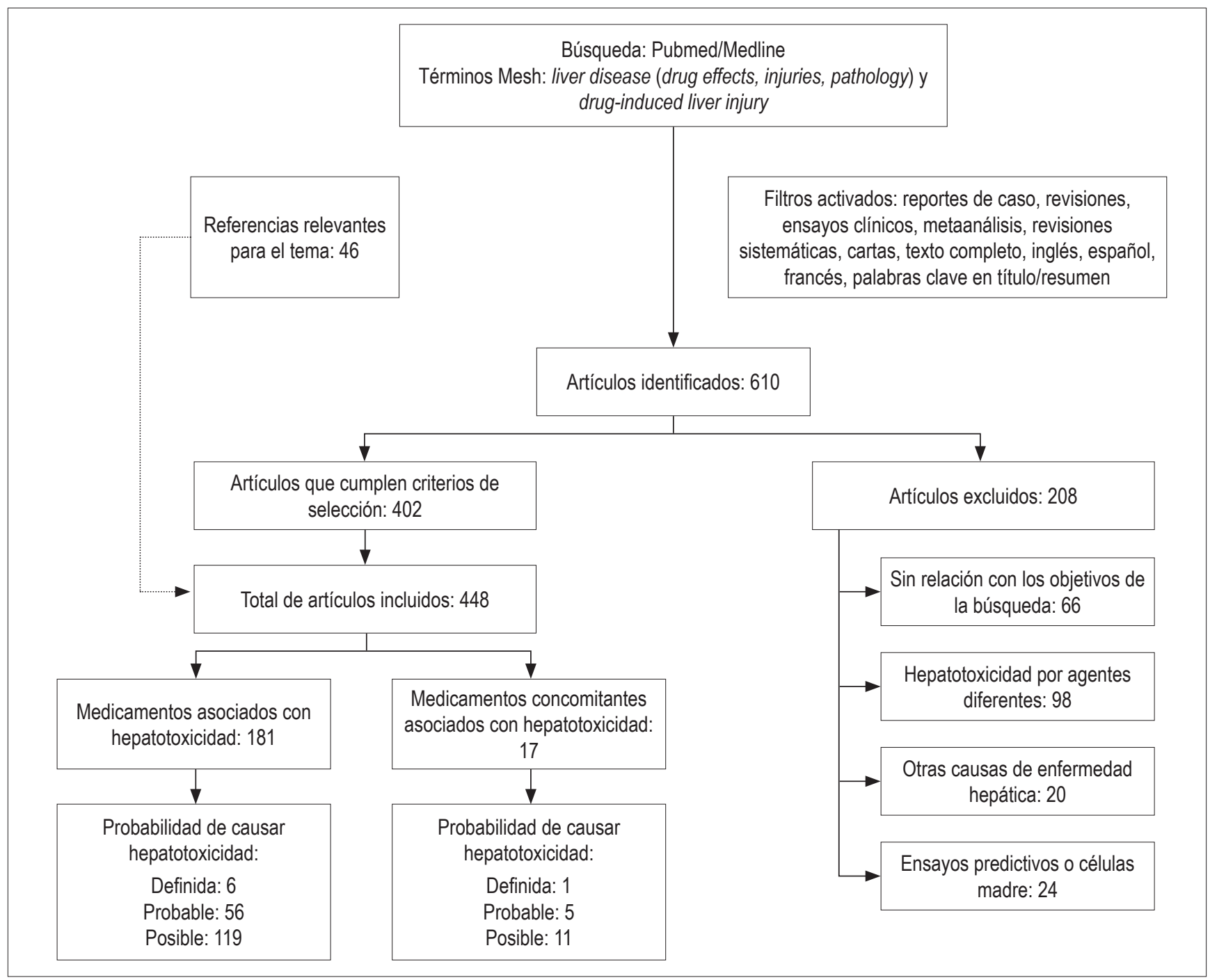

Figura 1. Resultados generales de la revisión estructurada: medicamentos causantes de toxicidad hepática.

mentos y 17 formas farmacéuticas combinadas o regímenes terapéuticos con probabilidad de causar hepatotoxicidad, de estos tuvieron probabilidad "definida" 6 medicamentos (metotrexato, minociclina, vancomicina, everolimus, isoniazida y tamoxifeno) y un régimen terapéutico (isoniazida, rifampicina más pirazinamida), 56 medicamentos y 5 formas farmacéuticas combinadas o regímenes terapéuticos "probable", 119 medicamentos y 11 formas farmacéuticas combinadas o regímenes terapéuticos "posible".

Se identificó el tipo de lesión (hepatocelular, colestásica y mixta) causado por cada fármaco, siendo la hepatocelular la más común. Los aspectos encontrados para cada medicamento con probabilidad definida (tipo de hepatotoxicidad, tipo de lesión y su aparición, mecanismo de hepatotoxicidad, factores de riesgo, manifestaciones clínicas y desenlaces) se tabularon (Tabla 1). Los fármacos encontrados se clasificaron según su grupo farmacológico y su código ATC para unificarlos; los fármacos con probabilidad probable se encuentran en la Tabla 2 y los fármacos con probabilidad posible se encuentran en la Tabla 3. Las cifras de enzimas hepáticas y dosis encontradas se hallan en la Tabla 4.

Entre los medicamentos antidiabéticos, se determinó la probabilidad (probable) de causar hepatotoxicidad de acarbosa $(42)$ y troglitazona $(43,44)$ (retirado del mercado); los reportes de caso publicaron lesiones de tipo hepatocelular, colestásica y mixta, acompañadas de ictericia, exantema y fiebre, entre otros. Los antiarrítmicos asociados fueron propafenona (45) y amiodarona, este último con más casos reportados y asociado con elevación de enzimas hepáticas en $15 \%-55 \%$ de los pacientes (46); la suspensión de los 
Tabla 1. Medicamentos con probabilidad de causar hepatotoxicidad definida

\begin{tabular}{|c|c|c|c|c|c|c|c|c|}
\hline $\begin{array}{c}\text { Grupo } \\
\text { farmacológico }\end{array}$ & $\begin{array}{l}\text { Fármaco } \\
\text { (cantidad de } \\
\text { artículos) } \\
\text { [código ATC] }\end{array}$ & $\begin{array}{l}\text { Evidencia de } \\
\text { hepatotoxicidad } \\
\text { (tipos de diseño } \\
\text { de estudio) }\end{array}$ & $\begin{array}{l}\text { Tipo de hepato- } \\
\text { toxicidad }\end{array}$ & $\begin{array}{l}\text { Tipo de lesión } \\
\text { (probabilidad } \\
\text { de aparición) }\end{array}$ & $\begin{array}{l}\text { Mecanismo de } \\
\text { hepatotoxicidad }\end{array}$ & Factores de riesgo & Manifestaciones clínicas & Manejo y desenlace \\
\hline Antibióticos & $\begin{array}{l}\text { Vancomicina (1) } \\
\text { [J01XA01] }\end{array}$ & $\begin{array}{l}\text { Definida (revisión } \\
\text { sistemática y } \\
\text { metaanálisis) }\end{array}$ & $\begin{array}{l}\text { Idiosincrásica } \\
\text { (inmunoalérgica) }\end{array}$ & $\begin{array}{l}\text { Hepatocelular } \\
\text { (definida) }\end{array}$ & $\begin{array}{l}\text { Toxicidad directa } \\
\text { o reacciones } \\
\text { adversas inmunes }\end{array}$ & Sin información & Elevación de aminotransferasas & Sin información \\
\hline Antibióticos & $\begin{array}{l}\text { Minociclina (5) } \\
\text { [J01AA08] }\end{array}$ & $\begin{array}{l}\text { Definida (revisión } \\
\text { sistemática, } \\
\text { reporte de } 2 \text { casos } \\
\text { clínicos y revisión } \\
\text { de grupos de } \\
\text { expertos) }\end{array}$ & Idiosincrásica & $\begin{array}{l}\text { Hepatocelular } \\
\text { (definida) }\end{array}$ & $\begin{array}{l}\text { Peroxidación } \\
\text { lipídica-necrosis }\end{array}$ & $\begin{array}{l}\text { Mujeres entre } 16 \text { y } 57 \text { años } \\
\text { con acné }\end{array}$ & $\begin{array}{l}\text { Hepatitis autoinmune. Elevación } \\
\text { de aminotransferasa, anticuerpos } \\
\text { anitinucleares positivos, ictericia, } \\
\text { anorexia, fiebre, artralgia, fatiga, } \\
\text { dolor abdominal, náuseas, exantema, } \\
\text { eosinofilia, inflamación periportal, } \\
\text { prurito. Hepatocitos hinchados y } \\
\text { colapsados. Esteatosis }\end{array}$ & $\begin{array}{l}\text { Trasplante de hígado, } \\
\text { muerte. Suspensión del } \\
\text { medicamento y evolución } \\
\text { favorable }\end{array}$ \\
\hline Antituberculosos & $\begin{array}{l}\text { Isoniazida (3) } \\
\text { [J04AC01] }\end{array}$ & $\begin{array}{l}\text { Definida (estudio } \\
\text { prospectivo y } \\
\text { retrospectivo, } \\
\text { y revisiones } \\
\text { de grupos de } \\
\text { expertos) }\end{array}$ & Idiosincrásica & $\begin{array}{l}\text { Hepatocelular } \\
\text { (probable) }\end{array}$ & $\begin{array}{l}\text { Peroxidación } \\
\text { lipídica-necrosis }\end{array}$ & $\begin{array}{l}\text { Mujeres con edad promedio } \\
\text { de } 60 \text { años y hombres } \\
\text { con edad promedio de } \\
54 \text {. Pacientes con edad } \\
\text { promedio de } 49 \text { años y } 70 \% \\
\text { de ellos mujeres }\end{array}$ & $\begin{array}{l}\text { Hepatitis aguda. Elevación de } \\
\text { aminotransferasas, náuseas, ictericia, } \\
\text { dolor abdominal, fatiga, anorexia, } \\
\text { vómito, orina oscura. Necrosis } \\
\text { hepatocelular }\end{array}$ & $\begin{array}{l}\text { Suspensión del } \\
\text { medicamento. Muerte, } \\
\text { trasplante hepático }\end{array}$ \\
\hline Antituberculosos & $\begin{array}{l}\text { Isoniazida + } \\
\text { rifampicina + } \\
\text { pirazimanida (4) } \\
\text { [J04AM05] }\end{array}$ & $\begin{array}{l}\text { Definida (revisión } \\
\text { sistemática } \\
\text { y revisiones } \\
\text { de grupos de } \\
\text { expertos) }\end{array}$ & Idiosincrásica & $\begin{array}{l}\text { Hepatocelular } \\
\text { (posible) }\end{array}$ & $\begin{array}{l}\text { Peroxidación } \\
\text { lipídica-necrosis. } \\
\text { Polimorfismo } \\
\text { genético, } \\
\text { formación de } \\
\text { metabolitos } \\
\text { hepatotóxicos }\end{array}$ & $\begin{array}{l}\text { Edad avanzada y sexo } \\
\text { femenino, coinfección con } \\
\text { VIH. Polimorfismo genético }\end{array}$ & $\begin{array}{l}\text { Elevación de enzimas hepáticas, } \\
\text { ictericia, dolor abdominal, náuseas, } \\
\text { vómito, astenia, esteatosis y necrosis. } \\
\text { Encefalopatía hepática, inflamación } \\
\text { granulomatosa }\end{array}$ & $\begin{array}{l}\text { Cambio o suspensión de } \\
\text { medicamento y evolución } \\
\text { favorable. Trasplante de } \\
\text { hígado }\end{array}$ \\
\hline Antiestrógeno & $\begin{array}{l}\text { Tamoxifeno (5) } \\
\text { [L02BA01] }\end{array}$ & $\begin{array}{l}\text { Definida (estudio } \\
\text { clínico controlado } \\
\text { aleatorizado y } \\
\text { reporte de } 6 \text { casos } \\
\text { clínicos) }\end{array}$ & Sin información & $\begin{array}{l}\text { Hepatocelular } \\
\text { (definida) }\end{array}$ & $\begin{array}{l}\text { Disminución de } \\
\text { la oxidación } \beta \text { de } \\
\text { ácidos grasos- } \\
\text { esteatohepatitis }\end{array}$ & $\begin{array}{l}\text { Mujeres de } 50 \text { y } 70 \\
\text { años con mastectomía, } \\
\text { diabetes, consumo } \\
\text { de alcohol <20 g/día, } \\
\text { histerectomía, sobrepeso, } \\
\text { hipercolesterolemia, } \\
\text { hipertensión, osteoporosis }\end{array}$ & $\begin{array}{l}\text { Esteatohepatitis, fibrosis, necrosis } \\
\text { hepatocelular, cirrosis micronodular, } \\
\text { hepatomegalia. Elevación de ALT. } \\
\text { Náuseas, vómito, malestar, infiltrado } \\
\text { inflamatorio. Dolor en cuadrante } \\
\text { superior derecho y peliosis hepática }\end{array}$ & $\begin{array}{l}\text { Evolución favorable. } \\
\text { Muerte. }\end{array}$ \\
\hline Antineoplásico & $\begin{array}{l}\text { Everolimus (1) } \\
\text { [L01XE10] }\end{array}$ & $\begin{array}{l}\text { Definida (revisión } \\
\text { sistemática y } \\
\text { metaanálisis) }\end{array}$ & Sin información & Sin información & Sin información & $\begin{array}{l}\text { Pacientes con neoplasias y } \\
\text { trasplantes de órganos }\end{array}$ & Elevación de ALT, fatiga & $\begin{array}{l}\text { Ajuste del tratamiento } \\
\text { o suspensión del } \\
\text { medicamento }\end{array}$ \\
\hline $\begin{array}{l}\text { Antineoplásico/ } \\
\text { inmunomodulador }\end{array}$ & $\begin{array}{l}\text { Metotrexato (7) } \\
\text { [L04AX03] }\end{array}$ & $\begin{array}{l}\text { Definida } \\
\text { (metaanálisis, } \\
\text { estudio } \\
\text { prospectivo, } \\
\text { estudio analítico) }\end{array}$ & Sin información & $\begin{array}{l}\text { Hepatocelular } \\
\text { (posible) }\end{array}$ & $\begin{array}{l}\text { Peroxidación } \\
\text { lipídica-necrosis. } \\
\text { Depleción } \\
\text { mitocondrial }\end{array}$ & $\begin{array}{l}\text { Niños con enfermedad } \\
\text { hepática previa. } \\
\text { Alcoholismo. Edad } \\
\text { adulta o avanzada. Dosis } \\
\text { acumuladas. Obesidad y } \\
\text { DM2 Esteroides. Exposición } \\
\text { previa a hepatotoxinas }\end{array}$ & $\begin{array}{l}\text { Elevación de enzimas hepáticas. } \\
\text { Fibrosis. Infiltrado inflamatorio. } \\
\text { Esteatohepatitis. Necrosis. Cirrosis }\end{array}$ & $\begin{array}{l}\text { Cambio de farmacoterapia. } \\
\text { Suspensión del } \\
\text { medicamento y evolución } \\
\text { favorable, muerte }\end{array}$ \\
\hline
\end{tabular}


Tabla 2. Medicamentos con probabilidad de causar hepatotoxicidad probable

\begin{tabular}{|c|c|c|c|c|}
\hline \multicolumn{5}{|c|}{ Medicamentos } \\
\hline Acarbosa & Acetaminofén & Alopurinol & Azatioprina & Ácido valproico \\
\hline Amiodarona & Benzarona & Aurotiomalato de sodio & Buprenorfina & Ciprofloxacina \\
\hline Atorvastatina & Clorhidrato de doxapram & Bentazepam & Etopósido & Clometiazol \\
\hline Enalapril & Efavirenz & Carbamazepina & Flutamida & Dextropropoxifeno \\
\hline Ezetimiba & Fluconazol & Ciproterona acetato & Glatiramer & Flucloxacilina \\
\hline Flupirtina & Itraconazol & Dantroleno & Imatinib & Lamotrigina \\
\hline Metildopa & Ketoconazol & Diclofenaco & Infliximab & Nitrofurantoína \\
\hline Papaverina & Metilprednisolona & Halotano & Ipilimumab & Ornidazol \\
\hline Perhexilina & Nevirapina & Isoflurano & Oxaliplatino & Telitromicina \\
\hline Propafenona & Propiltiouracilo & Lumiracoxib & Temozolomida & Tolcapona \\
\hline Troglitazona & Rifampicina & Nimesulida & Tioguanina & Trovafloxacina \\
\hline Vitamina A (retinol) & & & & \\
\hline
\end{tabular}

Tabla 3. Medicamentos con probabilidad de causar hepatotoxicidad posible

\begin{tabular}{|c|c|c|c|c|c|}
\hline \multicolumn{6}{|c|}{ Medicamentos } \\
\hline Ácido tióctico & Alfuzosina & Amodiaquina & Amoxicilina & Ácido nicotínico & Ácido acetilsalicílico \\
\hline Actinomicina D & Carmustina & Clozapina & Anfotericina B & Ácido tienílico & Alendronato sódico \\
\hline Busulfán & Ciclofenil & Enflurano & Atomoxetina & Ajmaline & Aurotioglucosa \\
\hline Donepezilo & Ciclofosfamida & Felbamato & Brivudina & Bosentan & Bromfenaco sódico \\
\hline Esomeprazol & Citarabina & Fenitoína & Cefdinir & Candesartan & Etretinato \\
\hline Glibenclamida & Dacarbazina & Fenobarbital & Clindamicina & Clopidogrel & Glucosamina \\
\hline Gliclazida & Disulfiram & Imipramina & Dapsona & Dabigatran & Ibuprofeno \\
\hline Glimepirida & Leflunomida & Mefloquina & Daptomicina & Dalteparina & Indometacina \\
\hline Loratadina & Mitoxantrona & Metilfenidato & Didanosina & Diltiazem & Mebendazol \\
\hline Mesalamina & Naftidrofuril & Metoxiflurano & Doxiciclina & Dipirona & Metimazol \\
\hline Mesalazina & Pazopanib & Mirtazapina & Eritromicina & Fenprocumón & Metronidazol \\
\hline Metformina & Pravastatina & Nefazodona & Espiramicina & Fosinopril & Nafamostat \\
\hline Nilutamida & Raloxifeno & Nomifensina & Espironolactona & Fumarato ferroso & Octreótido \\
\hline $\mathrm{N}$-óxido de indicina & Simvastatina & Propofol & Fosfomicina & Hidralazina & Oxaprozina \\
\hline Orlistat & Sirolimus & Quetiapina & Levofloxacina & Irbesartán & Piroxicam \\
\hline Oximetolona & Talidomida & Risperidona & Nafcilina & Labetalol & Rofecoxib \\
\hline Pioglitazona & Tamsulosina & Sertralina & Oxacilina & Lisinopril & Sitaxentán \\
\hline Ranitidina & Tocilizumab & Sevoflurano & Quinina & Nicardipino & Terbinafina \\
\hline Rosiglitazona & Trabectedina & Tiopental & Sulfadimetoxina & Quinidina & \\
\hline Sulfasalazina & $\begin{array}{l}\text { Undecanoato de } \\
\text { testosterona }\end{array}$ & Zolmitriptán & Zidovudina & Ticlopidina & \\
\hline
\end{tabular}

medicamentos se asoció con la mejoría de los pacientes, aunque existen reportes de muerte con amiodarona (47). Los antihipertensivos como enalapril elevaron las cifras de enzimas hepáticas y produjeron ictericia y cambios estructurales en el hígado confirmados por biopsia, que llevaron al trasplante y muerte (48). Para metildopa (probable) se identificaron 9 reportes de casos de toxicidad hepática de tipo idiosincrásico (17), con un patrón de lesión hepatocelular especialmente en mujeres, manifestado por ictericia, anorexia y náuseas; además, la biopsia hepática identificó necrosis e infiltrado inflamatorio $(49,50)$. Con atorvastatina (51) y ezetimiba (52), el tipo de lesión identificado fue hepatocelular, acompañada de elevación de enzimas hepáticas, ictericia, fiebre y astenia. 
Tabla 4. Valores de enzimas hepáticas y dosis asociadas con medicamentos hepatotóxicos

\begin{tabular}{|c|c|c|c|c|c|c|c|c|c|c|c|}
\hline \multirow[t]{2}{*}{ Fármaco } & \multicolumn{2}{|c|}{ AST (U/L) } & \multicolumn{2}{|c|}{ ALT (U/L) } & \multicolumn{2}{|c|}{$\mathrm{FA}(\mathrm{U} / \mathrm{L})$} & \multicolumn{2}{|c|}{ BT (mg/dL) } & \multicolumn{3}{|c|}{ Dosis } \\
\hline & Prom & $\mathrm{DE}$ & Prom & $\mathrm{DE}$ & Prom & $\mathrm{DE}$ & Prom & $\mathrm{DE}$ & Prom & $\mathrm{DE}$ & Unidad \\
\hline Acetaminofén & 10432,00 & 9533,8 & 2780,33 & 2471,0 & 247,78 & 122,5 & 6,78 & 4,6 & 4,38 & 1,3 & g/día \\
\hline Diclofenaco & 259,80 & 238,5 & 491,86 & 424,5 & 294,67 & 129,0 & 10,6 & 0 & 135,7 & 37,8 & $\mathrm{mg} / \mathrm{día}$ \\
\hline Buprenorfina & 1023,6 & 1016,2 & 2301 & 2455,2 & 216,75 & 74,9 & 86,67 & 93,6 & 10 & 4 & $\mathrm{mg} / \mathrm{día}$ \\
\hline Isoflurano & 7342,0 & 12129,7 & 5963,5 & 6265,7 & 176 & 110,0 & 9,55 & 5,5 & - & - & - \\
\hline Amiodarona & 359,9 & 523,4 & 716,00 & 867,4 & 208,67 & 128,2 & 3,0 & 2,3 & 528,57 & 226,8 & $\mathrm{mg} / \mathrm{día}$ \\
\hline Propafenona & 106 & 109,1 & 111,67 & 96,0 & 791,33 & 418,4 & 6,79 & 2,9 & 375 & 106,1 & $\mathrm{mg} / \mathrm{día}$ \\
\hline Nitrofurantoína & 652,5 & 717,7 & 690,5 & 291,5 & 214,1 & 112,0 & 9,6 & 9,9 & 144,4 & 110,2 & $\mathrm{mg} / \mathrm{día}$ \\
\hline Ornidazol & 1027,4 & 543,9 & 1315,4 & 1018,5 & 509,6 & 353,2 & 23,3 & 17,6 & 1000,0 & 0,0 & $\mathrm{mg} / \mathrm{día}$ \\
\hline Telitromicina & 1209 & 1663,1 & 1008,25 & 862,0 & 281 & 215,8 & 115,2 & 103,0 & 800 & 0 & $\mathrm{mg} / \mathrm{día}$ \\
\hline Minociclina & 833,83 & 689,1 & 1090 & 830,7 & 132,20 & 77,5 & 6,82 & 9,7 & 140,88 & 108,5 & $\mathrm{mg} / \mathrm{día}$ \\
\hline Ciprofloxacina & 360 & 260,6 & 520 & 210,7 & 308,67 & 288,8 & 3,77 & 1,8 & 833,33 & 288,7 & $\mathrm{mg} / \mathrm{día}$ \\
\hline Carbamazepina & 276,40 & 376,7 & 92 & 0 & 275,3 & 110,1 & 4,7 & 5,8 & 433,3 & 331,7 & $\mathrm{mg} / \mathrm{día}$ \\
\hline Lamotrigina & 1824 & 2716,7 & 2578,3 & 4061,4 & 147 & 33,9 & 13,07 & 2,3 & 75 & 0 & $\mathrm{mg} / \mathrm{día}$ \\
\hline Ácido valproico & 3196 & 4911,6 & 1361,3 & 1169,5 & 238,7 & 44,2 & 7,0 & 6,4 & 1050 & 320,2 & $\mathrm{mg} / \mathrm{día}$ \\
\hline Itraconazol & 189 & 181,1 & 177,7 & 79,0 & 1276 & 143,0 & 11 & 6,6 & 233,3 & 152,8 & $\mathrm{mg} / \mathrm{dí} a$ \\
\hline Alopurinol & 373,7 & 225,5 & 839,7 & 861,3 & 225,67 & 28,9 & 3,45 & 2,8 & 260,0 & 89,4 & $\mathrm{mg} / \mathrm{día}$ \\
\hline Enalapril & 849 & 461,9 & 689,25 & 537,8 & 542,75 & 180,4 & 10,525 & 6,3 & 15 & 5,8 & $\mathrm{mg} / \mathrm{día}$ \\
\hline Etopósido & 1540 & 177,8 & 1476,7 & 745,7 & 212 & 59,0 & 170 & 84,1 & 155,7 & 82,5 & $\mathrm{mg} / \mathrm{día}$ \\
\hline Temozolomida & 387,75 & 363,3 & 719,75 & 722,9 & 364,25 & 160,3 & 8,4 & 6,5 & 180 & 24,5 & $\mathrm{mg} / \mathrm{día}$ \\
\hline Tolcapona & 2947,5 & 1563,0 & 1551,3 & 1296,3 & 176,33 & 112,0 & 11,4 & 13,0 & 166,7 & 57,7 & $\mathrm{mg} / \mathrm{día}$ \\
\hline Propiltiouracilo & 639,17 & 557,3 & 784,80 & 506,2 & 520,6 & 520,7 & 19,1 & 11,5 & 316,7 & 103,3 & $\mathrm{mg} / \mathrm{día}$ \\
\hline Rifampicina & - & - & 450,67 & 82,2 & 231 & 146,4 & 103,33 & 12,5 & 300 & 259,8 & $\mathrm{mg} / \mathrm{día}$ \\
\hline Atorvastatina & 638,3 & 503,7 & 480,7 & 443,7 & 495,7 & 88,9 & 60,6 & 13,5 & 40 & 34,6 & $\mathrm{mg} / \mathrm{dí}$ \\
\hline Infliximab & 893,20 & 1042,6 & 720,6 & 638,3 & 250,5 & 46,0 & 11,267 & 9,2 & 4,33 & 1,0 & g/día \\
\hline
\end{tabular}

ALT: Alanino amino trasferasa; AST: aspartato amino trasferasa; BT: Bilirrubina total; DE: desviación estándar; FA: Fostatasa alcalina; prom: promedio.

El propiltiouracilo causó la muerte de un paciente, afectó a mujeres y niñas, generando síntomas como ictericia, prurito y pérdida de peso; se encontraron en las biopsias hepáticas necrosis, fibrosis, infiltrado inflamatorio y ductopenia. La suspensión del medicamento favoreció la evolución de algunas pacientes (53). Con metilprednisolona, se identificaron 4 casos con aumento en los valores de las enzimas hepáticas, debilidad e ictericia en los pacientes, que mejoraron con la interrupción del medicamento (54).

Entre los antibióticos, con la vancomicina (55) y minociclina (56) se identificaron reacciones de tipo idiosincrásico $(17,33)$; la minociclina afectó a mujeres entre los 16 y 57 años con diagnóstico de hepatitis autoinmune. Por su parte, rifampicina causó lesiones hepatocelulares y afectó especialmente a mujeres $(57,58)$. Los siguientes antibióticos se clasificaron como probables causantes de hepatotoxicidad: nitrofurantoína (frecuencia de casos de $12 \%$ y de tipo idiosincrásico) $(59,60)$, flucoxacilina (11 casos de tipo idiosincrásico) (61), telitromicina (lesión hepatoce- lular con elevación de transaminasas y fiebre) (62), ciprofloxacina y trovafloxacina (retirada del mercado). En general, los desenlaces variaron desde una evolución favorable hasta trasplante de hígado o muerte del paciente.

Para el caso de los antimicóticos itraconazol, fluconazol y ketoconazol se identificó daño hepático que mejoró con la interrupción de los medicamentos (63-66). Los agentes antirretrovirales pueden causar hepatotoxicidad dependiente de la dosis, en especial los inhibidores de la transcriptasa reversa análogos de nucleósidos y los inhibidores de proteasa (67). Se reportaron casos con efavirenz y nevirapina, con elevación de transaminasas y una incidencia entre $1 \%$ y $14 \%$ (9). La coinfección por virus de la hepatitis B o C puede aumentar la hepatotoxicidad con los tratamientos antirretrovirales $(68,69)$.

La quimioterapia ha aumentado la esperanza de vida; sin embargo, su utilización puede causar alteraciones hepáticas en los pacientes (70), que pueden ir desde esteatosis y esteatohepatitis hasta cirrosis (71). En el caso del 
tamoxifeno, everolimus y metotrexato, la probabilidad fue definida. Medicamentos como flutamida, etopósido, imatinib, ipilimumab, oxaliplatino, temozolomida, tioguanina, glatiramer, azatioprina e infliximab se clasificaron con una probabilidad de causar hepatotoxicidad como probable.

Los AINE se identificaron como un grupo importante que puede causar alteraciones hepáticas $(1,72)$ principalmente de tipo idiosincrásico (73), en casos de abuso o sobredosis (74). Algunos factores de riesgo identificados fueron la edad, género femenino, consumo crónico de alcohol, fármacos concomitantes, enfermedades subyacentes, obesidad, DM2 y resistencia a la insulina (72). Algunos agentes causantes son el diclofenaco, lumiracoxib y nimesulida. Con acetaminofén, reconocido ampliamente como sustancia hepatotóxica intrínseca debido a un metabolito que causa necrosis hepática, se identificaron 14 reportes de casos caracterizados por lesiones hepatocelulares manejadas con $\mathrm{N}$-acetilcisteína y prednisona $(75,76)$, con mejoría de los pacientes.

El halotano fue el anestésico general con mayor probabilidad de causar toxicidad hepática. La predisposición genética, dosis repetidas, obesidad y edad avanzada fueron algunos factores de riesgo. Las mujeres tienen mayor probabilidad de sufrir un daño hepático con estos medicamentos con lesiones de tipo hepatocelular, aumento en las enzimas hepáticas, necrosis, fiebre, ictericia y fatiga (77-79).

En el caso de anticonvulsivantes, con ácido valproico se identificó un mayor número de casos de hepatotoxicidad (de tipo hepatocelular) y se manifestó con elevación de transaminasas, dolor abdominal, ictericia y anorexia; además, en las biopsias hepáticas se identificaron esteatosis microvesicular y macrovesicular, necrosis e infiltrado inflamatorio. Este medicamento puede causar lesiones hepáticas en menores de 30 años $(80,81)$. Con carbamazepina se identificaron casos, principalmente de tipo mixto y con formación de granulomas $(82,83)$. La lamotrigina generó casos de hepatotoxicidad idiosincrásica que requirieron trasplante hepático $(84,85)$.

Se encontraron 17 formas farmacéuticas combinadas o regímenes terapéuticos (usados simultáneamente) capaces de causar lesión hepatocelular de tipo idiosincrásico, como en el caso de isoniazida, rifampicina y pirazinamida (probabilidad definida). El evento se manifestó con elevación de enzimas hepáticas, dolor abdominal, ictericia, astenia, náuseas, vómito y necrosis; confirmada por biopsias hepáticas (86-88). En el caso de las formas farmacéuticas combinadas de antibióticos, como trimetoprima/sulfametoxazol y amoxicilina/ácido clavulánico, se identificaron casos de toxicidad hepática, de tipo idiosincrásico y clasificada como probable (57). El evento se presentó principalmente en hombres, causando ictericia y prurito; en algunos casos de amoxicilina/ácido clavulánico, el desenlace fue trasplante hepático o muerte (89). El esquema antirretroviral ritonavir, indinavir, darunavir y fosamprenavir se asoció con lesión hepática de tipo hepatocelular, acompañada de necrosis $(68,90)$. El reporte de casos de antineoplásicos (6-tioguanina, daunomicina y citosina arabinosa) usados como parte del régimen terapéutico para la leucemia mieloide en niños causó hepatomegalia, cirrosis y enfermedad veno-oclusiva (91).

\section{DISCUSIÓN}

Los antibióticos, antineoplásicos y antituberculosos fueron los grupos de medicamentos identificados con mayor probabilidad de causar hepatotoxicidad, acorde con los resultados de otras revisiones realizadas previamente $(5,23,33$, 57). Relacionado con los antibióticos, de vancomicina y minociclina se identificó evidencia suficiente de su capacidad de generar hepatotoxicidad idiosincrásica $(17,33)$ y un tipo de lesión hepatocelular $(33,55,56,92)$, la cual se valoró como definida. Por su parte, en el caso de otros antibióticos, en la tetraciclina se identificó su capacidad de generar daño hepático, manifestado como esteatohepatitis (93); pero la búsqueda no arrojó información suficiente para su inclusión.

Los reportes de caso permitieron identificar al tamoxifeno, everolimus y metotrexato como agentes que pueden causar daño en el hígado, con una probabilidad definida. En el caso del metotrexato, un estudio publicado previamente (94), aunque reportó aumento en las enzimas hepáticas, no identificó correlación entre las concentraciones de metotrexato y la probabilidad de causar hepatotoxicidad. Con la información encontrada no fue posible establecer el tipo de hepatotoxicidad para estos 3 medicamentos; sin embargo, el daño tiende a ser de tipo hepatocelular, con elevación en los valores de transaminasas y con un desenlace que puede ir desde la evolución favorable hasta la muerte.

En esta revisión no se encontraron reportes de casos específicos de hepatotoxicidad por isoniazida; aunque, algunos autores afirman que este medicamento causa daño hepático (95). Así, es importante destacar que el uso concomitante de isoniazida, rifampicina y pirazinamida se identificó como causante de una hepatotoxicidad de tipo idiosincrásica y una lesión de tipo hepatocelular $(86,87)$, generando la suspensión de la farmacoterapia.

En esta revisión, para el acetaminofén, identificado como causante de toxicidad hepática, se valoró su probabilidad como probable, debido a que se identificaron solo opiniones de grupos de expertos y reportes de caso. Por esto, la ausencia de evidencia en metaanálisis, revisiones sistemáticas o ensayos clínicos no permitió su clasificación en la probabilidad definida.

En el caso de amiodarona, existen dudas sobre el tipo de hepatotoxicidad que genera. Aunque se encontró infor- 
mación que soporta reacciones de tipo idiosincrásico (32, 96-98), también se reportaron reacciones de tipo intrínseco, secundario al depósito del fármaco en el tejido hepático (23).

Existen medicamentos que han sido retirados del mercado mundial o de ciertos países por estar asociados con la probabilidad de causar hepatotoxicidad (99), algunos de estos identificados en esta revisión son el dextropropoxifeno, ketoconazol (aún comercializado en Colombia), nefazodona, propofol y sitaxentán.

La elevación de las enzimas hepáticas estuvo presente en muchos de los reportes de caso, convirtiéndose en un marcador a considerar en caso de sospecha de hepatotoxicidad por medicamentos. En este marco se presentaron las cifras de ingreso de ALT, AST, FA y BT para algunos medicamentos, tomadas de los diferentes reportes de caso; además, se mostraron los datos disponibles de las dosis administradas, información que podría apoyar la hipótesis de la aparición del evento a dosis terapéuticas.

Esta revisión tiene como limitación el uso de solo una base de datos (Pubmed/Medline) para la búsqueda de la información, lo que puede disminuir el número de medicamentos con probabilidad de causar toxicidad hepática e influir en la valoración de la probabilidad encontrada de los medicamentos tabulados. Para disminuir el riesgo de sesgos de información se usó el procedimiento propuesto por Amariles y colaboradores (41) tomando como base la probabilidad de aparición u ocurrencia de hepatotoxicidad, la que se estableció en 3 categorías (definida, probable y posible) de acuerdo con los tipos de estudios publicados (nivel de evidencia) para cada fármaco; y para disminuir el sesgo de confusión entre los medicamentos que se usaron al mismo tiempo, se hace la diferencia entre las formas farmacéuticas combinadas (varios fármacos en una sola forma farmacéutica) y los regímenes terapéuticos.

\section{CONCLUSIONES}

Se identificaron más de 180 medicamentos asociados con hepatotoxicidad, de los cuales 6 tienen una probabilidad definida; mientras que en su mayoría la probabilidad es posible. Se aprecia que más del $50 \%$ de los medicamentos encontrados se asocia con hepatotoxicidad de tipo idiosincrásico y el sexo femenino con un amplio rango de edad como principal factor de riesgo. Además, la elevación de las enzimas hepáticas, la ictericia y la fiebre hacen parte de los síntomas que más se presentan, generándose lesiones principalmente de tipo hepatocelular, seguidas de necrosis hepática. En la mayoría de los casos, se presenta una adecuada evolución al identificar y suspender el agente causante. La consolidación de la información sobre hepatotoxicidad demostró que diversos grupos de medicamen- tos tienen mayor evidencia de ser sustancias causantes de toxicidad hepática, aunque para algunas sea escasa la información precisa del mecanismo que explica el desarrollo y aparición del evento.

\section{Fuente de apoyo financiero}

El grupo Promoción y Prevención Farmacéutica recibió financiación de la convocatoria de sostenibilidad 2014-2015 del Comité para el Desarrollo de la Investigación (CODI) de la Universidad de Antioquia, Medellín, Colombia.

\section{Conflicto de intereses}

Los autores manifiestan que no presentan ningún conflicto de interés.

\section{REFERENCIAS}

1. Ibáñez L, Pérez E, Vidal X, et al. Prospective surveillance of acute serious liver disease unrelated to infectious, obstructive, or metabolic diseases: epidemiological and clinical features, and exposure to drugs. J Hepatol. 2002;37(5):592600. https://doi.org/10.1016/S0168-8278(02)00231-3

2. Fernández-Castañer A, García-Cortés M, Lucena $M$, et al. An analysis of the causes, characteristics, and consequences of reexposure to a drug or compound responsible for a hepatotoxicity event. Rev Esp Enferm Dig. 2008;100(5):278-84.

3. Lee W. Drug-Induced Hepatotoxicity. $\mathrm{N}$ Engl J Med. 2003;349(5):474-85. https://doi.org/10.1056/NEJMra021844

4. Chen M, Borlak J, Tong W. High lipophilicity and high daily dose of oral medications are associated with significant risk for drug-induced liver injury. Hepatology. 2013;58(1):38896. https://doi.org/10.1002/hep.26208

5. Tejada F. Hepatotoxicidad por fármacos. Rev clínica Med Fam. 2010;3(3):177-91.

6. Sgro C, Clinard F, Ouazir K, et al. Incidence of drug-induced hepatic injuries: A French population-based study. Hepatology. 2002;36(2):451-5. https://doi.org/10.1053/ jhep.2002.34857

7. Martí L, OlmoJ, Tosca J, et al. Clinical evaluation of drug-induced hepatitis. Rev Esp Enfermedades Dig. 2005;97(4):25865. https://doi.org/10.4321/S1130-01082005000400006

8. Hernández N, Bessone F, Sánchez A, et al. Profile of idiosyncratic drug induced liver injury in Latin America. An analysis of published reports. Ann Hepatol. 2014;13(2):231-9.

9. Liss G, Lewis J. Drug-induced liver injury: what was new in 2008? Expert Opin Drug Metab Toxicol. 2009;5(8):84360. https://doi.org/10.1517/17425250903018904

10. Ghabril M, Fontana R, Rockey D, et al. Drug induced liver injury caused by intramuscular administered medications: the drug induced liver injury network (DILIN) experience. J Clin Gastroenterol. 2013;47(6):553-8. https://doi. org/10.1097/MCG.0b013e318276bf00 
11. Larrey D. Epidemiology and individual susceptibility to adverse drug reactions affecting the liver. Semin Liver Dis. 2002;22(2):145-55. https://doi.org/10.1055/s-2002-30101 https://doi.org/10.1055/s-2002-30105

12. Ramachandran R, Kakar S. Histological patterns in druginduced liver disease. J Clin Pathol. 2009;62(6):481-92. https://doi.org/10.1136/jcp.2008.058248

13. Fisher K, Vuppalanchi R, Saxena R. Drug-induced liver injury. Arch Pathol Lab Med. 2015;139(7):876-87. https:/ / doi.org/10.5858/arpa.2014-0214-RA

14. Castell J, Miñana M. Hepatitis inducida por tóxicos. Mecanismos de toxicidad y patrones de lesión. GH Contin. 2003;2(5):190-6.

15. Lee WM. Drug induced hepatotoxicity. N Engl J Med. 1995;333(17):1118-27. https:// doi.org/ 10.1056/ NEJM199510263331706

16. Kaplowitz N. Drug-induced liver injury. Clin Infect Dis. 2004;38(Suppl 2):44-8. https://doi.org/10.1086/381446

17. Adams D, Ju C, Ramaiah S, et al. Mechanisms of immunemediated liver injury. Toxicol Sci. 2010;115(2):307-21. https://doi.org/10.1093/toxsci/kfq009

18. Grattagliano I, Bonfrate L, Diogo C, et al. Biochemical mechanisms in drug-induced liver injury: Certainties and doubts. World J Gastroenterol. 2009;15(39):4865-76. https://doi.org/10.3748/wjg.15.4865

19. García-Cortés M, Andrade R, Lucena $M$, et al. Hepatotoxicidad secundaria a fármacos de uso común. Gastroenterol Hepatol. 2005;28(8):461-72. https://doi. org/10.1157/13079002

20. Tarantino G, Di Minno M, Capone D. Drug-induced liver injury: Is it somehow foreseeable? World J Gastroenterol. 2009;15(23):2817-33. https://doi.org/10.3748/wjg.15.2817

21. Bakke O, Manochia M, De Abajo F, et al. Drug safety discontinuations in the United Kingdom, the United States, and Spain from 1974 through 1993: a regulatory perspective. Clin Pharmacol Ther. 1995;58(1):108-17. https://doi. org/10.1016/0009-9236(95)90078-0

22. Bénichou C. Criteria of drug-induced liver disorders. Report of an international consensus meeting.J Hepatol. 1990;11(2):2726. https://doi.org/10.1016/0168-8278(90)90124-A

23. Kaplowitz N. Drug Induced - Hepatotoxicity. Ann Intern Med. 1986;104(6):826-39. https://doi.org/10.7326/00034819-104-6-826

24. Reuben A. Hy’s Law. Hepatology. 2004;39(2):574-8. https://doi.org/10.1002/hep.20081

25. Björnsson E. Drug-induced liver injury: Hy's rule revisited. Clin Pharmacol Ther. 2006;79(6):521-8. https://doi. org/10.1016/j.clpt.2006.02.012

26. Pessayre D, Fromenty B, Berson A, et al. Central role of mitochondria in drug-induced liver injury. Drug Metab Rev. 2012;44(1):34-87. https://doi.org/10.3109/03602532.20 11.604086

27. Pessayre D, Mansouri A, Berson A, et al. Mitochondrial involvement in drug-induced liver injury. Handb Exp Pharmacol. 2010;(196):311-65. https://doi.org/10.1007/978-3-64200663-0_11
28. Kass G, Price S. Role of mitochondria in drug-induced cholestatic injury. Clin Liver Dis. 2008;12(1):27-51. https:// doi.org/10.1016/j.cld.2007.11.005

29. Labbe G, Pessayre D, Fromenty B. Drug-induced liver injury through mitochondrial dysfunction: mechanisms and detection during preclinical safety studies. Fundam Clin Pharmacol. 2008;22(4):335-53. https://doi.org/10.1111/ j.1472-8206.2008.00608.x

30. Amacher D. A toxicologist's guide to biomarkers of hepatic response. Hum Exp Toxicol. 2002;21(5):253-62. https:// doi.org/10.1191/0960327102ht247oa

31. Brunt E. Nonalcoholic steatohepatiiis (NASH): further expansion of this clinical entity? Liver. 1999;19(4):263-4. https://doi.org/10.1111/j.1478-3231.1999.tb00047.x

32. Gunawan B, Kaplowitz N. Clinical perspectives on xenobioticinduced hepatotoxicity. Drug Metab Rev. 2004;36(2):30112. https://doi.org/10.1081/DMR-120034148

33. Hayashi P, Fontana R. Clinical features, diagnosis, and natural history of drug-induced liver injury. Semin Liver Dis. 2014;34(2):134-44. https://doi. org/10.1055/s-0034-1375955

34. Hewitt M, Enoch S, Madden J, et al. Hepatotoxicity: a scheme for generating chemical categories for read-across, structural alerts and insights into mechanism(s) of action. Crit Rev Toxicol. 2013;43(7):537-58. https://doi.org/10.3 109/10408444.2013.811215

35. Kleiner D. The pathology of drug-induced liver injury. Semin Liver Dis. 2009;29(4):364-72. https://doi. org/10.1055/s-0029-1240005

36. Regev A, Seeff L, Merz M, et al. Causality assessment for suspected DILI during clinical phases of drug development. Drug Saf. 2014;37(S1):S47-56. https://doi.org/10.1007/ s40264-014-0185-4

37. Lucena M, Camargo R, Andrade R, et al. Comparison of two clinical scales for causality assessment in hepatotoxicity. Hepatology. 2001;33(1):123-30. https://doi.org/10.1053/ jhep.2001.20645

38. Lucena M, Andrade R, Rodrigo L, et al. TrovafloxacinInduced Acute Hepatitis. Clin Infect Dis. 2000;30(2):4001. https://doi.org/10.1086/313680

39. Andrade R, López-Ortega S. Hepatitis tóxicas. Rev Española Enfermedades Dig. 2006;98(9):701. DOI: 10.4321/S113001082006000900009.

40. Björnsson E. Hepatotoxicity associated with antiepileptic drugs. Acta Neurol Scand. 2008;118(5):281-90. https:// doi.org/10.4321/S1130-01082006000900009

41. Amariles P, Giraldo N, Faus M. Interacciones medicamentosas: Aproximación para establecer y evaluar su relevancia clínica. Med Clin (Barc). 2007;129(1):27-35. https://doi. org/10.1157/13106681

42. Fujimoto Y, Ohhira M, Miyokawa N, et al. Acarbose-induced hepatic injury. Lancet. 1998;351(9099):340. https://doi. org/10.1016/S0140-6736(05)78337-9

43. Schiano TD, Bellary S V, Cassidy MJ, et al. Subfulminant liver failure and severe hepatotoxicity caused by loratadine 
use. Ann Intern Med. 1996;125(9):738-40. https://doi. org/10.7326/0003-4819-125-9-199611010-00006

44. Li H, Heller D, Leevy C, et al. Troglitazone-induced fulminant hepatitis. Report of a case withautopsy findings. J Diabetes Complications. 2000;14(3):175-7. https://doi. org/10.1016/S1056-8727(00)00076-3

45. Cocozzella D, Curciarello J, Corallini O, et al. Propafenone hepatotoxicity: report of two new cases. Dig Liver Dis. 2003;48(2):354-7.

46. Flaharty K, Chase S, Yaghsezian H, et al. Hepatotoxicity associated with amiodarone therapy. Pharmacotherapy. 1989;9(1):3944. https://doi.org/10.1002/j.1875-9114.1989.tb04102.x

47. Shepherd N, Dawson A, Crocker P, et al. Granular cells as a marker of early amiodarone hepatotoxicity: a pathological and analytical study. J Clin Pathol. 1987;40(4):418-23. https://doi.org/10.1136/jcp.40.4.418

48. Jeserich $\mathrm{M}$, Ihling $\mathrm{C}$, Allgaier $\mathrm{H}$, et al. Acute liver failure due to enalapril. Herz. 2000;25(7):689-93. https://doi. org/10.1007/PL00001983

49. Thomas E, RosenthalW,Zapiach L, et al. Spectrum of methyldopa liver injury. Am J Gastroenterol. 1977;68(2):125-33.

50. Puppala A, Steinheber F. Fulminant hepatic failure associated with methyldopa. Am J Gastroenterol. 1977;68(6):578-81.

51. Pelli N, Setti M, Ceppa P, et al. Autoimmune hepatitis revealed by atorvastatin. Eur J Gastroenterol Hepatol. 2003;15(8):921-4. https://doi.org/10.1097/00042737200308000-00014

52. Stolk M, Becx M, Kuypers K, et al. Severe hepatic side effects of ezetimibe. Clin Gastroenterol Hepatol. 2006;4(7):90811. https://doi.org/10.1016/j.cgh.2006.04.014

53. Jonas M, Eidson M. Propylthiouracil hepatotoxicity: two pediatric cases and review of literature. J Pediatr Gastroenterol Nutr. 1988;7(5):776-8. https://doi. org/10.1097/00005176-198809000-00027

54. Melamud B, Lurie Y, Goldin E, et al. Methylprednisoloneinduced liver injury: a diagnostic challenge. IMAJ. 2014;16(3):180-1.

55. Chen Y, Yang X, Zeckel M, et al. Risk of hepatic events in patients treated with vancomycin in clinical studies: a systematic review and meta-analysis. Drug saf. 2011;34(1):7382. https://doi.org/10.2165/11539560-000000000-00000

56. Lawrenson R, Seaman H, Sundström A, et al. Liver damage associated with minocycline use in acne: a systematic review of the published literature and pharmacovigilance data. Drug Saf. 2000;23(4):333-49. https://doi. org/10.2165/00002018-200023040-00006

57. Navarro V, Senior J. Drug-related hepatotoxicity. N Engl J Med. 2006;354(7):731-9. https://doi.org/10.1056/ NEJMra052270

58. Prince M, Burt A, Jones D. Hepatitis and liver dysfunction with rifampicin therapy for pruritus in primary biliary cirrhosis. Gut. 2002;50(3):436-9. https://doi.org/10.1136/ gut.50.3.436

59. Hydes T, Wright M, Jaynes E, et al. Nitrofurantoin immunemediated drug-induced liver injury: a serious complication of a commonly prescribed medication. BMJ Case Rep. 2014;2014. https://doi.org/10.1136/bcr-2013-203136

60. Edoute Y, Karmon Y, Roguin A, et al. Fatal liver necrosis associated with the use of nitrofurantoin. Isr Med Assoc J. 2001;3(5):382-3.

61. Koek G, Striker B, Blok A, et al. Flucloxacillin-associated hepatic iniurv. Liver. 1994;14(5):225-9. https://doi. org/10.1111/j.1600-0676.1994.tb00079.x

62. Clay K, Hanson J, Pope S, et al. Telithromycin: a possible cause of severe liver damage? Ann Intern Med. 2006; 144(6):I42.

63. Adriaenssens B, Roskams T, Steger P, et al. Hepatotoxicity related to itraconazole: report of three cases. Acta Clin Belg. 2001;56(6):364-9. https://doi.org/10.1179/acb.2001.055

64. Jacobson M, Hanks D, Ferrell L. Fatal acute hepatic necrosis due to fluconazole. Am J Med. 1994;96(2):188-90. https:// doi.org/10.1016/0002-9343(94)90141-4

65. Chien R, Yang L, Lin P, et al. Hepatic injury during ketoconazole therapy in patients with onychomycosis: a controlled cohort study. Hepatology. 1997;25(1):103-7. https://doi. org/10.1002/hep.510250119

66. Van Parys G, Evenepoel C, Van Damme B, et al. Ketoconazole-induced hepatitis: a case with a definite cause-effect relationship. Liver. 1987;7(1):27-30. https:// doi.org/10.1111/j.1600-0676.1987.tb00311.x

67. Akhtar M, Mathieson K, Arey B, et al. Hepatic histopathology and clinical characteristics associated with antiretroviral therapy in HIV patients without viral hepatitis. Eur J Gastroenterol Hepatol. 2008;20(12):1194-204. https:// doi.org/10.1097/MEG.0b013e328305b9e0

68. Macías J, Neukam K, Mallolas J, et al. Liver toxicity of initial antiretroviral drug regimens including two nucleoside analogs plus one non-nucleoside analog or one ritonavir-boosted protease inhibitor in $\mathrm{HIV} / \mathrm{HCV}$-coinfected patients. HIV Clin Trials. 2012;13(2):61-9. https://doi.org/10.1310/hct1302-61

69. Clarke S, Harrington P, Condon C, et al. Late onset hepatitis and prolonged deterioration in hepatic function assciated with nevirapine therapy. Int J STD AIDS. 2000;11(5):336-7. https://doi.org/10.1177/095646240001100511 https:// doi.org/10.1258/0956462001915831

70. McWhirter D, Kitteringham N, Jones R, et al. Chemotherapy induced hepatotoxicity in metastatic colorectal cancer: a review of mechanisms and outcomes. Crit Rev Oncol Hematol. 2013;88(2):404-15. https://doi.org/10.1016/j. critrevonc.2013.05.011

71. Choti M. Chemotherapy-associated hepatotoxicity: do we need to be concerned? Ann Surg Oncol. 2009;16(9):23914. https://doi.org/10.1245/s10434-009-0512-7

72. Agúndez J, Lucena M, Martínez C, et al. Assessment of nonsteroidal anti-inflammatory drug-induced hepatotoxicity. Expert Opin Drug Metab Toxicol. 2011;7(7):817-28. https://doi.org/10.1517/17425255.2011.574613

73. Boelsterli U, Zimmerman H, Kretz-Rommel A. Idiosyncratic liver toxicity of nonsteroidal antiinflammatory drugs: molecular mechanisms and pathology. Crit Rev Toxicol. 1995;25(3):20735. https://doi.org/10.3109/10408449509089888 
74. Prescott L. Hepatotoxicity of mild analgesics. Br J Clin Pharmacol. 1980;10(Suppl 2):373S-9S. https://doi. org/10.1111/j.1365-2125.1980.tb01825.x

75. Black M. Acetaminophen hepatotoxicity. Ann Rev Med. 1984;35:577-93. https:// doi.org/ 10.1146/ annurev.me. 35.020184.003045

76. Quartuccio L, Maset M, Soardo G, et al. Acetaminopheninduced liver injury in a woman with febrile flare of systemic lupus erythematosus. J Clin Rheumatol. 2014;20(6):349-51.

77. Miller D, Dwyer J, Klatskin G. Halothane hepatitis: benign resolution of a severelesion. Ann Intern Med. 1978;89(2):2125. https: / doi.org/10.7326/0003-4819-89-2-212

78. Munro H, Snider S, Magee J. Halothane-associated hepatitis in a 6-year-old boy: evidence for native liver regeneration following failed treatment with auxiliary liver transplantion. Anesthesiology. 1998;89(2):524-7. https://doi. org/10.1097/00000542-199808000-00033

79. Camilleri M, Victorino R, Hodgson H. Halothane aggravation of chronic liver disease. Acta Med Port. 1984;5(6):194-6.

80. Colleti R, Trainer T, Krawisz B. Reversible valproate fulminant hepatic failure. J Pediatr Gastroenterol Nutr. 1986;5(6):990-4. https://doi.org/10.1097/00005176198611000-00032

81. Bicknese A, May W, Hickey W, et al. Early childhood hepatocerebral degeneration misdiagnosed as valproate hepatotoxicity. Ann Neurol. 1992;32(6):767-75. https://doi. org/10.1002/ana.410320610

82. Levy M, Goodman M, Van Dyne B, et al. Granulomatous hepatitis secondary to carbamazepine. Ann Intern Med. 1981;95(1):64-5. https://doi.org/10.7326/0003-4819-95$1-64$

83. Horowitz S, Patwardhan R, Marcus E. Hepatotoxic reactions associated with carbamazepine therapy. Epilepsia. 1988;29(2):149-54.

84. Bhayana H, Appasami S, Thapa B, et al. Lamotrigineinduced vanishing bile duct syndrome in a child. J Pediatr Gastroenterol Nutr. 2012;55(6):e147-8. https://doi. org/10.1097/MPG.0b013e31823c2500

85. Mecarelli O, Pulitano P, Mingoia M, et al. Acute hepatitis associated with lamotrigine and managed with the molecular adsorbents recirculating system (MARS). Epilepsia. 2005;46(10):1687-9. https://doi.org/10.1111/j.15281167.2005.00269.x

86. Tostmann A, Boeree M, Aarnoutse R, et al. Antituberculosis drug-induced hepatotoxicity: concise up-to-date review. J Gastroenterol Hepatol. 2008;23(2):192-202. https://doi. org/10.1111/j.1440-1746.2007.05207.x

87. Cramer J, Lohse A, Burchard G, et al.Low N-acetyltransferase 2 activity in isoniazid-associated acute hepatitis requiring liver transplantation. Transpl Int. 2010;23(2):231-3. https://doi.org/10.1111/j.1432-2277.2009.00921.x
88. Yew W, Leung C. Antituberculosis drugs and hepatotoxicity. Respirology. 2006;11(6):699-707. https://doi. org/10.1111/j.1440-1843.2006.00941.x

89. Lucena M, Andrade J, Fernández M, et al. Determinants of the clinical expression of amoxicillin- clavulanate hepatotoxicity: a prospective series from Spain. Hepatology. 2006;44(4):850-6. https://doi.org/10.1002/hep.21324

90. Ortu F, Weimer L, Floridia M, et al. Raltegravir, tenofovir and emtricitabine in an HIV-infected patient with HCV chronic hepatitis, NNRTI intolerance and protease inhibitors-induced severe liver toxicity. Eur J Med Res. 2010;15(2):81-3.

91. D'Cruz C, Wimmer R, Harcke T, et al. Veno-occlusive disease of the liver in children following chemotherapy for acute myelocytic leukemia. Cancer. 1983;52(10):1803-7. https://doi. org/10.1002/1097-0142(19831115)52:10<1803::AIDCNCR2820521007>3.0.CO;2-D

92. Kaplowitz N. Idiosyncratic drug hepatotoxicity. Nat Rev Drug Discov. 2005;4(6):489-99. https://doi.org/10.1038/ nrd 1750

93. Schumacher J, Guo G. Mechanistic review of drug-induced steatohepatitis. Toxicol Appl Pharmacol. 2015;289(1):40-7. https://doi.org/10.1016/j.taap.2015.08.022

94. Fathi N, Mitros F, Hoffman J, et al. Longitudinal measurement of methotrexate liver concentrations does not correlate with liver damage, clinical efficacy, or toxicity during a 3.5 year double blind study in rheumatoid arthritis. J Rheumatol. 2002;29(10):2092-8.

95. Hayashi P, Fontana R, Chalasani N, et al. Under-reporting and poor adherence to monitoring guidelines for severe cases of isoniazid hepatotoxicity. Clin Gastroenterol Hepatol. 2015;13(9):1676-82. https://doi.org/10.1016/j. cgh.2015.02.024

96. Rigas B, Rosenfeld L, Barwick K, et al. Amiodarone hepatotoxicity. A clinicopathologic study of five patients. Ann Intern Med. 1986;104(3):348-51. https://doi. org/10.7326/0003-4819-104-3-348

97. Jain D, Bowlus C, James A, et al. Granular cells as a marker of early amiodarone hepatotoxicity. J Clin Gastroenterol. 2000;31(3):241-3. https://doi.org/10.1097/00004836200010000-00012

98. Lupon-Rosés J, Simó-Canonge R, Lu-Cortez L, et al. Probable early acute hepatitis with parenteral amiodarone. Clin Cardiol. 1986;9(5):223-5. https://doi.org/10.1002/ clc. 4960090512

99. Onakpoya I, Heneghan C, Aronson J. Post-marketing withdrawal of 462 medicinal products because of adverse drug reactions: a systematic review of the world literature. BMC Med. 2016;14(10):1-11. Ann Intern Med. 1986;104:34851. https://doi.org/10.1186/s12916-016-0553-2 